\title{
Entre paradigmas aristotélicos y galileanos, ¿Qué publican los(as) Trabajadores(as) Sociales chilenos(as)
}

\author{
Christian Quinteros Flores ${ }^{1}$ \\ https://orcid.org/0000-0002-6145-9347 \\ ${ }^{1}$ Universidad de Chile, Escuela de Gobierno y Gestiòn Pública, Santiago de Chile, Chile
}

\author{
Entre paradigmas aristotélicos y galileanos, ¿que publican los(as) trabajadores(as) sociales \\ chilenos(as)
}

Resumen: La evidencia indica que la disciplina del Trabajo Social se ha centrado históricamente en la intervención científica de lo social y ha descuidado lo puramente investigativo. Esta situación ha sido resultado probablemente de la existencia de distintas jerarquías disciplinares en el mundo científico. Sin embargo, frente a la crisis del modelo subsidiario, se han incrementado los espacios académicos para este tipo de producciones científicas. El presente artículo busca aproximarse a la forma en que los/as Trabajadores/ as Sociales chilenos(as) realizan sus procesos de producción y divulgación científica mediante un estudio de tipo exploratorio descriptivo de carácter documental. Se recogió información sobre paradigmas epistemológicos empleados, metodologías y técnicas de recolección de datos, entre otras dimensiones. Se observa una predominancia absoluta de los enfoques cualitativos en la producción científica y altas barreras de entrada para que profesionales puedan destinar tiempo y capacidades a la investigación, espacio que sigue estando fuertemente centralizado en la academia.

Palabras-clave: Epistemología de las Ciencias Sociales. Disciplina. Metodología de Investigación. Trabajo Social. Producción científica

\section{Between Aristotelian and Galilean paradigms: Which do Chilean social workers publish?}

Abstract: Evidence indicates that the discipline of Social Work has historically focused on the scientific intervention of social and has neglected the purely investigative. This situation has probably resulted from the existence of different disciplinary hierarchies in the scientific world. However, in the crisis of the subsidiary model, it has been increased academic spaces for this type of scientific production. This article seeks to approximate how Chilean Social Workers carry out their scientific production and dissemination processes through a descriptive exploratory study of documentary nature. Information collected on the epistemological paradigms used, methodologies, and data collection techniques, among other dimensions. There is an absolute predominance of qualitative approaches in scientific production and high barriers of entry so that professionals can allocate time and skills to research, this space that remains strongly centralized in the academy.

Keywords: Social Science Epistemology. Discipline. Research Methodology. Social Work. Scientific production.

Entre os paradigmas aristotélico e Galileu: o que publicam os assistentes sociais chilenos?

Resumo: As evidências indicam que a disciplina do Serviço Social historicamente se concentrou na intervenção científica do social e negligenciou o puramente investigativo. Essa situação provavelmente foi resultado da existência de diferentes hierarquias disciplinares no mundo científico. No entanto, diante da crise do modelo subsidiário, os espaços acadêmicos para esse tipo de produção científica aumentaram. Este artigo procura abordar a maneira como os assistentes sociais chilenos realizam seus processos de produção e divulgação científica por meio de um estudo exploratório descritivo de natureza documental. Foram coletadas informações sobre paradigmas epistemológicos utilizados, metodologias e técnicas de coleta de dados, entre outras dimensões. Existe uma predominância absoluta de abordagens qualitativas na produção científica e altas barreiras à entrada para que os profissionais possam dedicar tempo e recursos à pesquisa, um espaço que continua fortemente centralizado na academia.

Palavras-Chave: Epistemologia das Ciências Sociais, Disciplina, Metodologia da Pesquisa, Serviço Social, Produção científica.

Recebido em 08.11.2019. Aprovado em 11.02.2020. Revisado em 16.06.2020. 
que permite copiar, distribuir y reproducir en cualquier medio, así como también adaptar, transformar y crear a partir de este material, desde que para fines no comerciales, y que usted fornezca el crédito debido a los autores y a la fuente, insiera un enlace para la Licencia Creative Commons e indique si fueron hechas alteraciones. 


\section{Introducción}

En el siglo XVIII se produce en Europa, la irrupción de las denominadas ciencias del espíritu, humanas o sociales. Ante las crisis de la época, la sociedad requirió nuevas formas de comprender desde adentro los distintos fenómenos frente al fracaso de las ciencias naturales o formales en su intención de explicar la conducta humana y las nuevas dinámicas sociales. La sociedad requería mirarse a si misma y tomar conciencia de sí (Mardones y Ursua, 1982). De esta manera, aparecieron nuevas disciplinas como la psicología o la sociología, cuyos postulados de rigor científico incluso aún son cuestionados por algunos, planteándose el conocido problema epistemológico del criterio de demarcación de la ciencia. Por otra parte, el mundo científico se dividió entre aquellos científicos que adscribían a la tradición galileana de la ciencia y otros que lo hacían basados en concepciones aristotélicas de ella. Hoy, el Trabajo Social es interpelado como disciplina y profesión, a contribuir en la construcción de un nuevo orden social. Sin embargo, producto de políticas públicas basadas en estándares de eficiencia, sus profesionales destinan escaso tiempo a la producción de nuevo conocimiento científico. En este contexto, poco se sabe, por ejemplo, desde qué enfoque epistemológico observan la realidad estos investigadores, o que tan elitizada por la academia está esta práctica. El presente trabajo aborda parcialmente el criterio de demarcación de la ciencia, para luego describir la tensión epistemológica histórica entre las concepciones galileana y aristotélica y su contextualización de la práctica investigativa actual, para finalmente presentar al lector los resultados de la investigación.

\section{Marco Teórico}

Tal como apunta Padrón (2007), la noción de epistemología debe considerar diversas tendencias en su desarrollo histórico, y no puede ser tratada unívocamente ya que se deben considerar perspectivas de fondo que generan diversidades en los modos de hacer epistemología y de hablar de epistemología. Según este autor, existen variables con las que se puede clasificar los enfoques epistemológicos basadas en convicciones acerca de las fuentes del conocimiento (Gnoseológicas) o en convicciones acerca de las relaciones del sujeto con la realidad (Ontológicas). De esta manera, se pueden encontrar enfoques empiristas, racionalistas, idealistas y realistas. Este ciclo comprende desde una concepción de la 'ciencia de los objetos observables' (empirismorealista, Círculo de Viena, neopositivismo, neoconductismo...), pasando a una concepción de la 'ciencia de los objetos calculables' o 'pensables' (racionalismo-realista, Einstein, Popper), yendo luego a una concepción de la 'ciencia de los objetos intuibles' (racionalismo-idealista, hermenéutica, Teoría Crítica de Frankfurt...), hasta terminar en una concepción de la 'ciencia de los objetos vivibles' o 'experienciables' (empirismo idealista, etno metodología, investigación cualitativa (Padrón, 2007). Enfocándonos en el objeto de nuestro trabajo, es fundamental generar una base conceptual que permita comprender cuando hablamos de conocimiento fiable y/o conocimiento científico. En este sentido, consideraremos el problema de la demarcación entre lo que es científico y lo que no lo es, propuesto por Diéguez. Para este autor, y desde una perspectiva tradicional, los científicos investigan para alcanzar ciertos conocimientos acerca de la naturaleza y del hombre y que para ello observan, miden, experimentan, inventan teorías, se reúnen en congresos y publican en revistas especializadas (Diéguez, 2010). El conocimiento fiable, otorga el reconocimiento de la comunidad científica, el prestigio social y la-siempre complicada- asignación de recursos. ¿Pero cuál es ese conocimiento fiable?. No existe suficiente consenso al respecto. Para epistemólogos como Mario Bunge, donde no hay método científico no hay ciencia, ya que las normas metodológicas eliminarían la subjetividad del investigador y permitiría el consenso de la comunidad científica.

\section{Tabla 1 - Tipos de ciencia}

\begin{tabular}{l|l|l}
\hline Ciencias Formales & \multicolumn{2}{|c}{ Ciencias Empíricas } \\
\hline Lógica y matemáticas & $\begin{array}{l}\text { Ciencias naturales (física, química, } \\
\text { biología) } \\
\text { Análisis de causas }\end{array}$ & $\begin{array}{l}\text { Ciencias Humanas y sociales (sociología, economía y } \\
\text { psicología)Comprensión del significado de las acciones } \\
\text { humanas(tradición hermenéutica) }\end{array}$ \\
\hline
\end{tabular}

Fuente: Elaboración Propia a partir de Diéguez (2010). 
Sin embargo, autores como Feyerabend negaron la existencia de un método científico, ya que ninguno de ellos es de aplicación universal. Este autor reconocido crítico de la ortodoxia científica, planteó la postura del Todo vale, que correspondía más bien a una posición escéptica sobre la posibilidad de la demarcación y de diferenciar plenamente entre discursos científicos y no científicos. Sin el ánimo de profundizar en un debate que se aparte del objetivo de este trabajo, es importante contextualizar como se ha concebido la ciencia desde los enfoques galileanos, para poder comprender porque hoy se cuestionaría la calidad de los procedimientos cualitativos en la investigación social. Desde el círculo de Viena, se buscó establecer un rasgo diferenciador del conocimiento científico que fuera capaz de distinguirlo de otros productos culturales. Para ello plantearon criterios de demarcación: el principio de verificabilidad y el principio de confirmabilidad. La verificabilidad se refería a determinar que sería científico lo que tiene sentido de lo que no lo tiene. Un enunciado es significativo si se puede verificar en principio, aunque nadie haya logrado verificarlo. Por su parte la confirmabilidad poseía grados, las leyes pueden ser apoyadas crecientemente por enunciados observacionales. Las teorías científicas son susceptibles de encontrar experiencias que las apoyen. La falsabilidad “el tercer criterio de demarcación según Diéguez (2010) - se basa en el planteamiento positivista de Popper que señala que lo que distingue a la ciencia no es su capacidad para verificar o confirmar sus teorías sino, su capacidad para deshacerse rápidamente de las teorías erróneas mediante una crítica rigurosa orientada a la refutación de las teorías a partir de las experiencias. Una teoría corroborada significa que ha resistido los intentos de falsaciones. Interesan teorías arriesgadas ya que tienen más contenido empírico, por ejemplo, el enunciado Todas las aves del hemisferio norte son migratorias, anidan en la costa y son monógamas dice mucho más que todas las aves del hemisferio norte son migratorias ya que corresponde a un enunciado más informativo de la realidad y a la vez más arriesgado.

\section{Tradición Aristotélica V/S Tradición Galileana}

Entonces, el mundo científico occidental -incluidas las ciencias sociales " se ha desarrollado entre los avances de dos grandes tradiciones epistemológicas: la aristotélica y la galileana. La tradición aristotélica plantea que la observación del universo debe realizarse considerando un conjunto de sustancias, mediante un camino inductivo, donde preocupa la causa final (telos) de las cosas y el para qué. Le preocupa la sustancia de las cosas, sus propiedades, facultades, potencias asimismo como la explicación cualitativa de las cosas, donde el centro de todo es el mundo. Esta tradición busca básicamente Comprender los Hechos, y entender que la naturaleza enseña por lo que no es necesario experimentar con ella. Esta tradición - matriz de tradiciones hermenéuticas y fenomenológicas - se refiere a que los nuevos modos de organización social " producto de crisis sociales, de conflictos, de nuevos conceptos como el poder- exigen nuevas maneras de comprender la sociedad, y realizar una intervención consciente de la realidad. De esta manera, comprender representa una concepción metodológica propia de las ciencias humanas, que tiene resonancia psicológica, ya que sus métodos reactualizan la atmosfera espiritual, los sentimientos, los motivos, los valores de los sujetos. Desde esta tradición se postula una razón emancipadora para lograr una sociedad buena, humana y racional, donde no se puede desvincular el contexto de justificación (contexto sociopolítico) del contexto de descubrimiento. Este paradigma promueve un interés emancipador de la ciencia y la supresión de la injusticia social. (Mardones y Ursua, 1982).

Por su parte, la tradición Galileana y fundamentalmente su derivación positivista, se refiere a la Ciencia Moderna, que considera al universo como flujos de acontecimientos que se relacionan según leyes. Pregona una lógica funcional- mecanicista, basada en una explicación causal de los hechos. Esta tradición busca el poder y control sobre la naturaleza donde el centro es el Hombre. Para tal propósito, cosifica y reduce a objeto la naturaleza para sus necesidades y utilidades, lo que según Habermas (1981) podría denominarse la razón instrumental de la ciencia. Para Habermas, la racionalidad científica pertenece a un complejo de racionalidad cognitivo-instrumental que puede reclamar validez por encima del contexto de una cultura particular. Así la ciencia moderna, posee un interés pragmático y le preocupa el ¿Cómo? de las cosas, a través de leyes que relacionan numéricamente los fenómenos. Esta tradición promueve el análisis experimental, donde el investigador según se convierte en un "Juez que averigua lo que quiere". Horkheimer y Adorno no entienden el "dominio" sobre la naturaleza como una metáfora; bajo el concepto de "dominación" reducen a un común denominador el control sobre la naturaleza externa, el mando sobre los hombres y la represión de la naturaleza interna, de la propia naturaleza subjetiva: "El dominio sobre la naturaleza incluye el dominio sobre los hombres" (Habermas, 1981, Pág., 483) Corbetta (2007) señala que la "epistemología positivista" indica que los resultados son "ciertos" y objetivos, y establece la separación entre el observador y lo observado, predomina el uso de técnicas cuantitativas y el análisis por variables. Por otra parte, la "metodología interpretativa" de tradición aristotélica, establece una interacción empática entre investigador y el objeto de investigación y el uso de técnicas cualitativas (Corbetta, 2007). 


\section{El problema de la disociación entre investigación e intervención en Trabajo Social}

¿Qué sucede hoy en Trabajo Social? La respuesta a esta pregunta requiere la consideración de nuevos enfoques epistemológicos y nuevos contextos sociopolíticos, como lo es la crisis del estado subsidiario. Desde algunas posiciones críticas, se plantea que la lógica científica dominante (el paradigma galileano), establece rígidas separaciones en la clasificación de la ciencia y distingue entre ciencias puras, ciencias aplicadas y tecnologías, estableciendo una relación jerárquica entre ellas, donde se ha equiparado el quehacer del Trabajo Social a una tecnología social y el/la profesional a un sujeto bien entrenado para instrumentar las decisiones de otros en política social y que privilegia la racionalidad de la acción (Linardelli y Pessolano, 2019). Esta situación conspiraría frente al desarrollo de esta línea de acción del Trabajo Social, que requeriría fortalecer y/o ampliar el campo disciplinar con nuevas teorías, enfoques y hallazgos. Según Iturrieta, el concepto de profesión estaría ligado al de disciplina, ya que estas son las que producen y alimentan los conocimientos y se asocian a la idea de profesión en la medida que un caso de producción de conocimientos, se profesionaliza vía la materialización de tales conocimientos en fines prácticos y definidos. (Iturrieta, 2016). Un primer problema del Trabajo Social en la línea de investigación, es según Linardelli y Pessolano (2019), que al interior del campo científico y académico existen relaciones de hegemonía/subalternidad entre las diferentes disciplinas y áreas de conocimiento, como también entre diversas tradiciones teóricas. En ese contexto, la disciplina de Trabajo Social históricamente ha ocupado un lugar subalterno o subsidiario en el conjunto de la producción de saber de las ciencias sociales, identificándose como una profesión de orientación fundamentalmente interventiva. (Linardelli y Pessolano, 2019). Por otra parte, para Enríquez (2016), la reforma a la educación superior realizada en Chile en los años 80 trajo consigo la diversificación de las escuelas de Trabajo Social generando una multiplicidad de programas existentes en la formación de pregrado. Esta apertura originó un pluralismo formativo (Enríquez, 2016). A esto habría que agregar la masificación de las alternativas educacionales en Trabajo Social. Las prácticas investigativas de los y las trabajadores(as) sociales acompañan el quehacer profesional desde sus inicios, aunque desde la lógica de la intervención, lo que hoy no es suficiente para fortalecer su campo disciplinar propio, lo que genera una tensión no resuelta por la formación de pregrado. Segun Linarddelli y Pessolano (2019), las divisiones entre intervenir e investigar, puede explicarse en primer término, por la historia de constitución disciplinar del Trabajo Social latinoamericano, en la existe una tardía vinculación con el campo de las ciencias sociales. En segundo término, por el tipo de legitimidad socio-ocupacional obtenida por el Trabajo Social en tanto profesión, ubicada predominante en la intervención social y en tercer lugar, por la mayoría femenina en Trabajo Social, pues el campo científico ha sido asociado desde la modernidad con los rasgos estereotipados masculinos. Para otros, la influencia y contexto del sistema neoliberal- como dirían los aristotélicos asociados a la escuela Frankfurt al considerar que es imposible no considerar el socio histórico en los descubrimientos - han definido nuevas formas de hacer ciencia. Según Muñoz (2018), nos enfrentamos a una era en que la racionalidad neoliberal ha colonizado la forma de producción de conocimientos a escala global y por supuesto el Trabajo Social no ha estado exento de estas tensiones. La investigación ha sido identificada como una dimensión fundante de Trabajo Social. Según Toledo (2004), en las últimas décadas se ha debatido arduamente sobre las posibilidades y méritos del Trabajo Social para optar al reconocimiento de su carácter disciplinar, lo que - a juicio de sus defensores - vendría a superar las justificaciones ideológicas y tecnológicas. También ha estado en juego su definición como carrera profesional de rango estrictamente universitario. Ambas aspiraciones se han articulado en torno a un renovado interés por otorgar un lugar de relevancia al conocimiento científico en su quehacer, incorporando acciones de sistematización, investigación y construcción de teoría (Toledo, 2004). Tanto es así, que Trabajadoras/es Sociales que han completado estudios doctorales y/o que lideran proyectos de investigación financiados por la Comisión Nacional de Investigación Científica y Tecnológica (CONICYT) ha aumentado durante la última década (Rubilar, citado en Muñoz, 2018). Según Linardelli, y Pessolano (2019) en el campo del conocimiento, la ciencia moderna entrega el monopolio de la distinción entre lo verdadero y lo falso, en detrimento de formas alternativas de conocimientos (populares, campesinos, indígenas) que desaparecen como conocimientos relevantes porque se encuentran más allá de lo verdadero y lo falso. Frente a ello, plantea un pensamiento postabismal, compuesto por una ecología de saberes, que se caracteriza por afirmar la pluralidad de conocimientos existentes (entre los cuales se encuentra la ciencia moderna) y las conexiones continuas y dinámicas entre distintas formas de saber. No niega el saber científico, sino que propone un uso contra hegemónico del mismo. Nuevos enfoques son necesarios. Arellano y Castro (2017) plantean que propuestas pertinentes al interior del Trabajo Social para hacer frente a la contingencia de la crisis social han quedado acotadas al uso de teorías y metodologías de las ciencias sociales, mientras que las humanidades quedan en la periferia de la discusión. Creen que la filosofía e historia pueden aportar, dando algunas luces para el Trabajo Social y su acción en el contexto actual. Para Mosquera (2006), el reto que tenemos hoy es romper la tradicional dicotomía intervención e investigación social, apoyados en las nuevas armaduras conceptuales de una corriente del pensamiento posmoderno. 
Tabla 2 - Tendencias Epistemológicas en Trabajo Social

\begin{tabular}{l|l|l}
\hline \multicolumn{1}{c|}{ Primera tendencia } & \multicolumn{1}{c|}{ Segunda tendencia } & \multicolumn{1}{c}{ Tercera tendencia } \\
\hline $\begin{array}{l}\text { Predominio de una relación de dependencia } \\
\text { respecto a disciplinas del core knowledge, } \\
\text { o disciplinas fundamentales. (Trabajo So- } \\
\text { cial es una ciencia aplicada, encargada de la } \\
\text { solución de problemas y de la prestación } \\
\text { de servicios sociales). }\end{array}$ & $\begin{array}{l}\text { Adhesión a un modelo científico empírico- } \\
\text { deductivo en la intervención-investigación } \\
\text { en Trabajo Social, vista como otra vía hacia } \\
\text { la cientificidad. }\end{array}$ & $\begin{array}{l}\text { Comienzo de un movimiento de } \\
\text { reflexividad, en el cual se valorizan los sa- } \\
\text { beres de acción }\end{array}$ \\
\hline
\end{tabular}

Fuente: Elaboración propia a partir de Mosquera (2006).

\section{Metodología}

Este estudio de tipo descriptivo exploratorio se llevó a cabo mediante la revisión de 113 artículos de investigación publicados en las siete revistas chilenas de Trabajo Social. Se consideraron publicaciones especializadas asociadas a universidades donde se imparte la carrera, tanto del sector público o tradicionales como privadas, Como criterio de selección de las unidades de análisis se consideraron los trabajos investigativos donde al menos había colaborado como autor un (a) Trabajador(a) social chileno (a). Se consideraron las siguientes categorías: epistemología utilizada, técnicas de recolección de datos utilizadas, tamaño de la muestra o sujetos entrevistados, palabras claves y perfil del autor o autores. Como hipótesis de trabajo se planteó que las publicaciones científicas tendrían una mayor relación con enfoques cualitativos, dada la comentada categorización científica histórica del Trabajo Social y a la predominancia de nuevas epistemologías en el quehacer interventivo. Los objetivos del trabajo son:1. Identificar los paradigmas epistemológicos y las metodologías de investigación que subyacen en cada publicación; 2. Precisar las técnicas de recolección de datos utilizadas en las investigaciones, 3 . Determinar las áreas de mayor interés que abordan los artículos, 4.Conocer el perfil del investigador responsable de cada trabajo.

Tabla 3 - Revistas analizadas

\begin{tabular}{|c|c|c|c|c|}
\hline Revista & Universidad & Indexación & $\begin{array}{c}\text { Números } \\
\text { analizados }\end{array}$ & $\begin{array}{l}\text { Año de } \\
\text { inicio }\end{array}$ \\
\hline Electrónica de Trabajo Social & Universidad de Concepción & LATINDEX & 30 & 1998 \\
\hline Rumbos & Universidad Central de Chile & $\begin{array}{l}\text { ERIH P, LATINDEX-Directorio y Ca- } \\
\text { tálogo 1.0, REDIB, CLASE -Asociación } \\
\text { de Revistas de Humanidades y Ciencias } \\
\text { Sociales, LATINREV }\end{array}$ & 26 & 2006 \\
\hline Trabajo Social & $\begin{array}{l}\text { Pontificia Universidad Católica } \\
\text { de Chile }\end{array}$ & & 15 & 1970 \\
\hline Cuadernos de Trabajo Social & $\begin{array}{l}\text { Universidad Tecnológica Metro- } \\
\text { politana UTEM }\end{array}$ & LATINDEXZ DirectorioEBSCO & 14 & 2002 \\
\hline $\begin{array}{l}\text { Perspectivas: Notas sobre } \\
\text { intervención y acción social }\end{array}$ & $\begin{array}{l}\text { Universidad Católica Silva } \\
\text { Henríquez }\end{array}$ & $\begin{array}{l}\text { DIALNET } \\
\text { LATINDEX. Catálogo v2.0 }\end{array}$ & 14 & 1995 \\
\hline Intervención & Universidad Alberto Hurtado & LATINDEX & 9 & 2011 \\
\hline $\begin{array}{l}\text { Pensamiento y Acción } \\
\text { Interdisciplinaria }\end{array}$ & Universidad Católica del Maule & LATINDEX DirectorioDIALNET & 5 & 2016 \\
\hline
\end{tabular}

Fuente: Elaboración propia(2020). 


\section{Resultados}

La investigación evidenció un amplio abordaje del paradigma interpretativo lo que significó un 89\% de las publicaciones analizadas, lo que comprueba la hipótesis planteada en esta investigación. Por su parte los estudios realizados desde la perspectiva cuantitativa alcanzaron un $6 \%$, mientras que los enfoques mixtos un $5 \%$. Este aspecto se explicaría debido a la mayor familiarización que tienen los Trabajadores Sociales con enfoques más comprensivos, que como se planteó en el marco teórico buscan conocer desde adentro las subjetividades de los sujetos, desde sus propias narrativas y comprensiones del mundo. Esta situación debe estar relacionada además con los procesos formativos y académicos recibidos por los investigadores.

Figura 1 - Investigaciones según Paradigma Epistemológico utilizado

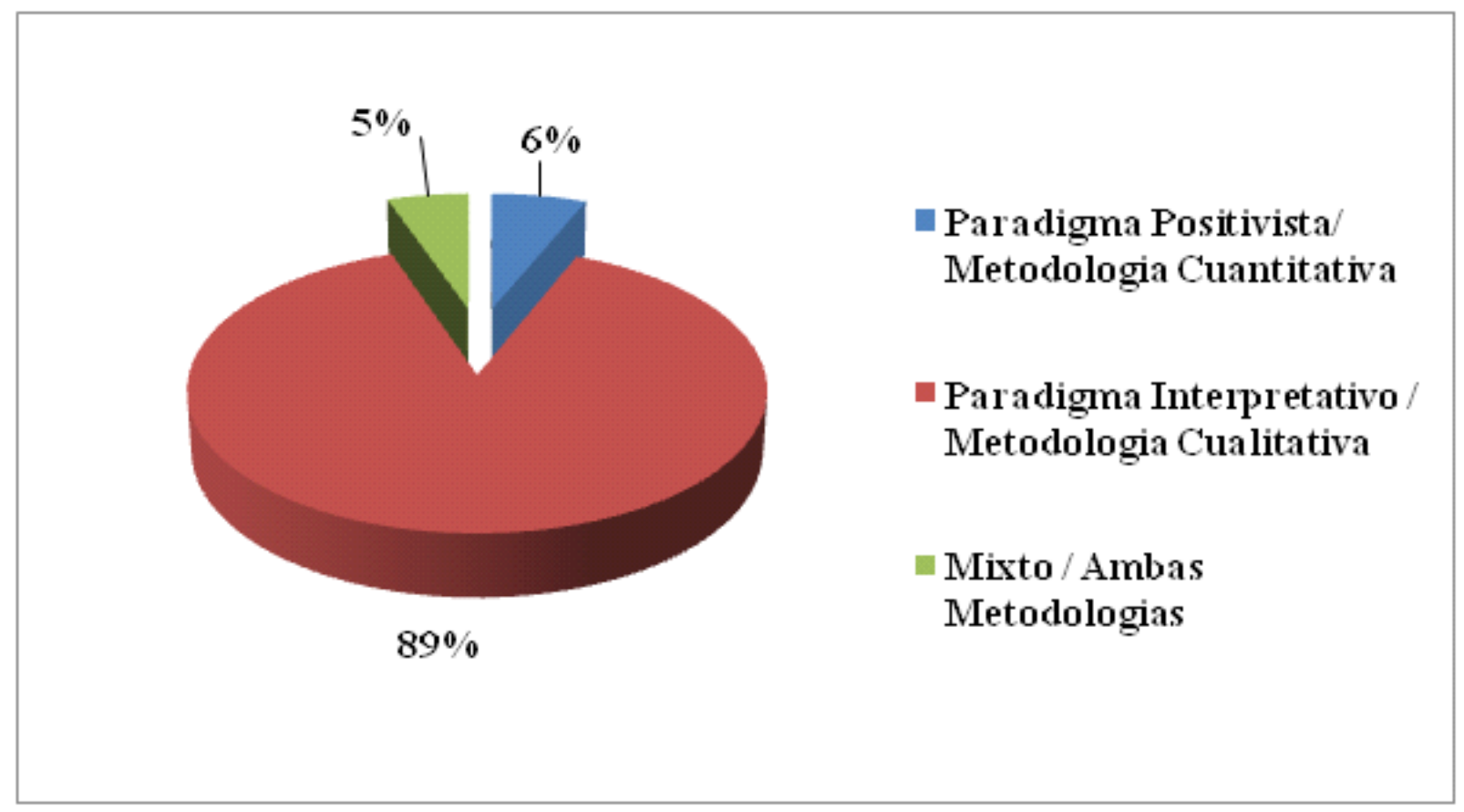

Fuente: Elaboración Propia(2020).

Dentro de las técnicas de recolección de datos, llama la atención el alto uso de la técnica conocida como Revisión Bibliográfica que llega a casi 30 investigaciones (26\%) analizadas. Esto se podría explicar, por un lado, por el alto costo que significa realizar un proceso de investigación empírico, que levante muestras o unidades de análisis, según sea el paradigma escogido. La revisión bibliográfica supone análisis de textos, de teorías, de triangulaciones entre autores. Como segunda preferencia de técnicas aparece la "Entrevista semi estructurada", ya sea como única herramienta investigativa o complementada con otras como el análisis documental, la observación participante o las historias de vida. El "análisis documental" aparece como la tercera opción utilizada en estas publicaciones, referida básicamente a revisiones de leyes, normativas institucionales, actas de reuniones, tesis de grado, entre otras. La sistematización de procesos es también utilizada por algunos(as) investigadores (as), referidas a integración de otras investigaciones, procesos cualitativos desarrollados en algunos proyectos de intervención, u otros. Las entrevistas grupales y los grupos de discusión (focus groups) también aparecen en la constatación de estas evidencias. En el caso de las investigaciones cuantitativas, predomina la revisión de base de datos estadísticos, con utilización de indicadores, pero solo desde una perspectiva exploratoria y descriptiva. No se registró ninguna investigación que pretendiera cruzar variables o contrastar hipótesis. Esto podría estar asociado a la baja habilitación de este tipo de instrumentos en la fase formativa de los trabajadores sociales en el pre grado y a la falta de apropiación en el uso de estadígrafos o software estadísticos como el SPSS, ninguna de las investigaciones analizadas consideró este tipo de herramientas. En un segundo lugar, aparecen las encuestas /cuestionario, en su gran mayoría aplicadas en los contextos institucionales donde se desempeñaban los profesionales. 
Figura 2 - Técnicas de Recolección de Datos Utilizadas

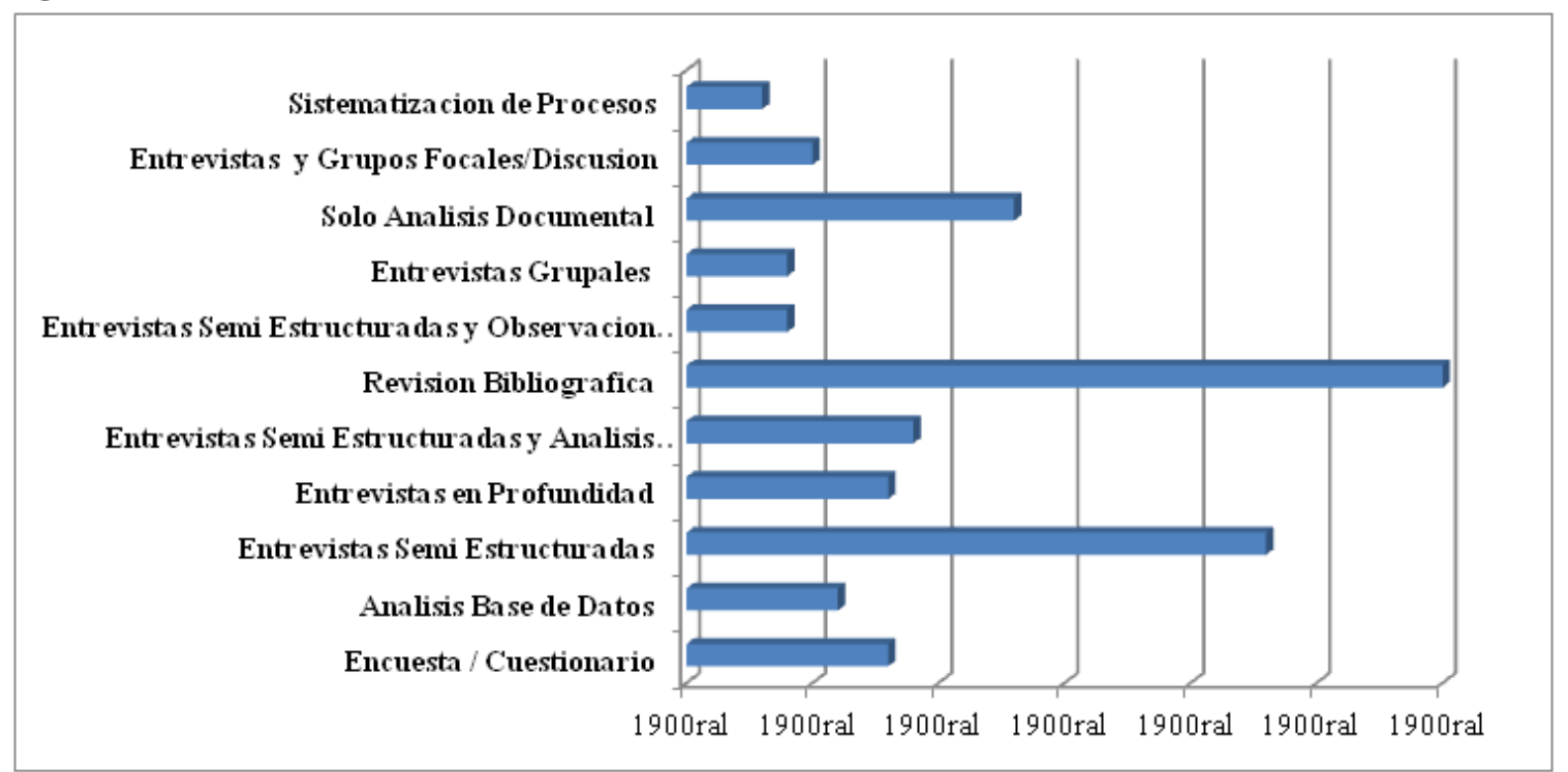

Fuente: Elaboración Propia(2020).

Respecto de los alcances o coberturas de las investigaciones de tipo cualitativas, llama la atención el alto número de ellas que consideró una muestra menor a 10 sujetos (24). En el rango de 11 a 20 se registraron 14 de ellas. Este es probablemente una limitación que ofrece este tipo de estudios cualitativos que precisamente al no buscar "verdades" científicas, se dedican a comprender subjetividades individuales y colectivas, lo que para las lógicas institucionales de financiamiento de investigaciones reducen su impacto al momento de intentar concluir sobre generalidades de ciertos comportamientos sociales. Probablemente esta situación es la que genera una mirada de reojo hacia este tipo de investigaciones por parte de la institucionalidad científica que sigue priorizando investigaciones que permitan un mayor grado de generalizaciones. El denominado prestigio social que permite la asignación de recursos según Dieguez (2010).

Figura 3 - Número de Sujetos Entrevistados en cada investigación (Cualitativa)

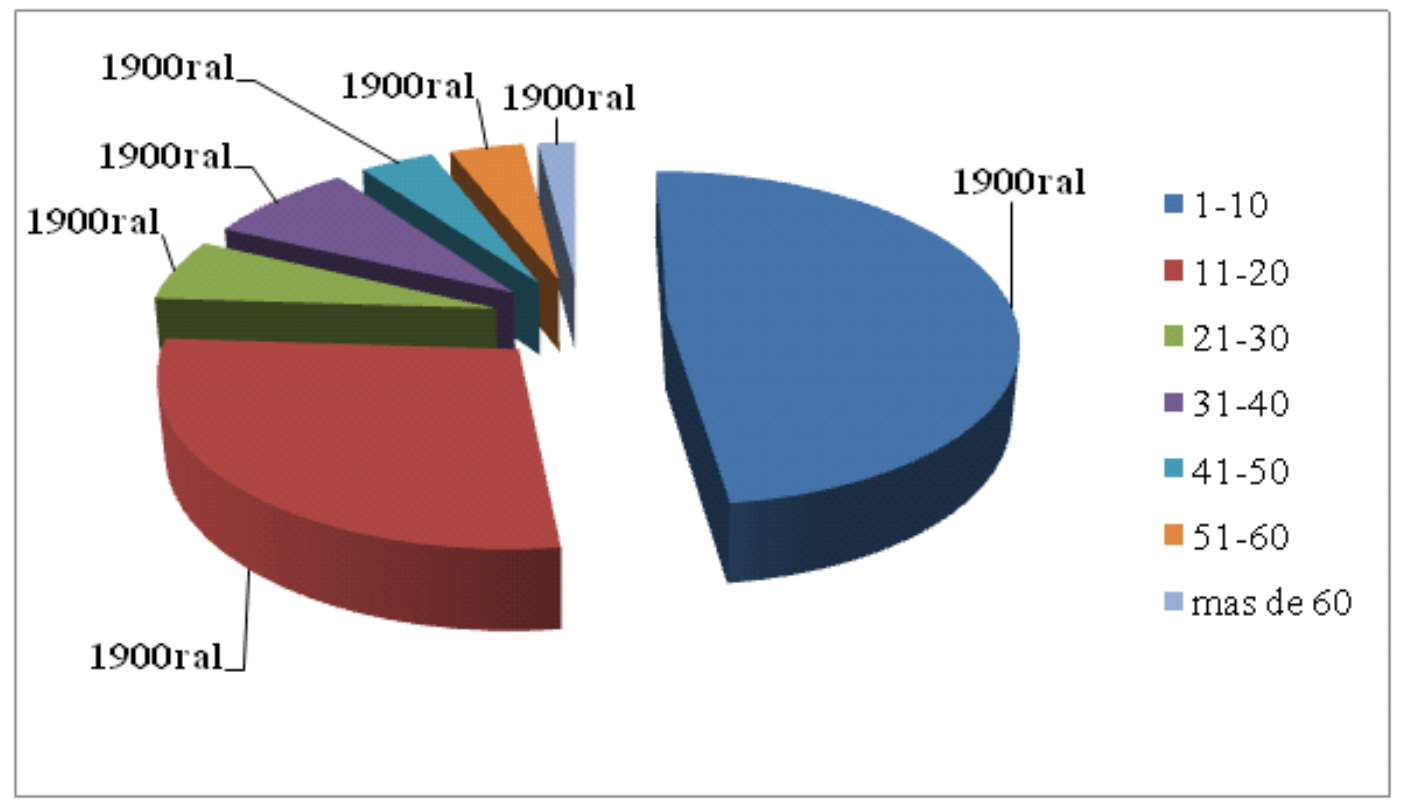

Fuente: Elaboración Propia(2020). 
Respecto del perfil de los y las Trabajadores(as) Sociales que realizaron las investigaciones, se constata que un $78 \%$ de ellos están relacionados con la academia, ya sea, como encargado de núcleos académicos de investigación, como jefes de carrera, o en calidad de docente, mientras que solo un $22 \%$ corresponde a profesionales vinculados a instituciones como ONGs, hospitales u oficinas municipales. Esta situación demuestra una brecha importante en el desarrollo de los procesos investigativos, ya que son aun escasos los profesionales que se dedican a generar nuevos conocimientos, independiente del paradigma o método que utilicen, ya que la academia- como es de esperar- asume con mayor vigor esta tarea, lo que debe llevar a replantear la necesidad de potenciar este tipo de espacios. Aquí se advierte un desafío urgente de la política pública, la de confiar a los Trabajadores y Trabajadoras Sociales una mayor dedicación a este tipo de acciones. Probablemente el desafío para los investigadores está localizado en ampliar las unidades de análisis o las muestras, para contribuir con mayor alcance a las políticas públicas y de considerar paralelamente la consideración de otras formas de conocer, que buscan comprender más que explicar. Esta tensión no es una cuestión particular del Trabajo Social sino de la mayoría de las ciencias humanas o sociales. (Figura 4)

\section{Figura 4 - Perfil de Investigadores}

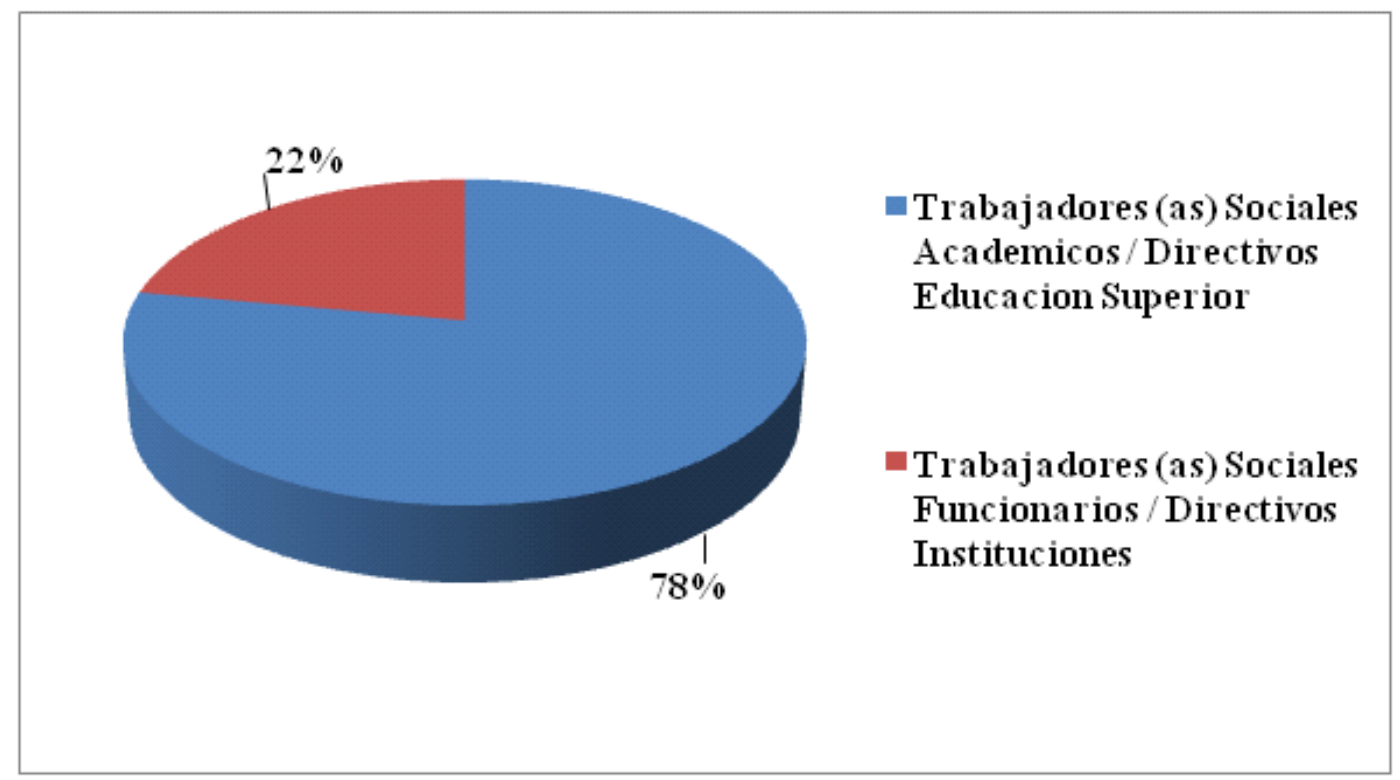

Fuente: Elaboración propia(2020).

Finalmente, las principales áreas temáticas abordada por los y las Trabajadores Sociales están referidas principalmente a las políticas públicas y/o sociales, a su focalización y a sus estrategias, desde una mirada más bien crítica de sus supuestos, resultados o metodologías. Siguen en preferencias, las preocupaciones por los asuntos de la sociedad civil, donde esta se considera como un actor relevante en nuevos escenarios de gobernanza territorial. En tercer lugar, destacan las investigaciones de asuntos de género, de feminización de los aspectos públicos, asociadas a cuestiones de equidad social y de trato igualitario entre hombres y mujeres. En cuarto lugar, aparece la preocupación por los asuntos relacionados con la familia, sin profundizar en su tipología. La preocupación por la salud pública es un tema que también capta la atención de los y las investigadoras, específicamente en la evaluación de programas y en la calidad de la atención primaria. También se ubican en un lugar relevante el interés de los investigadores con los asuntos propios de la intervención profesional, de la calidad de los diagnósticos, de las evaluaciones, de los procesos de sistematización, seguidos en orden descendente por los asuntos de derechos humanos, territorio, identidad, y violencia intrafamiliar, principalmente (Figura 5)

\section{¿Qué sucede hoy en Trabajo}

Social? La respuesta a esta pregunta requiere la consideración de nuevos enfoques epistemológicos y nuevos contextos sociopolíticos, como lo es la crisis del estado subsidiario. 
Figura 5 - Principales Áreas Temáticas abordadas

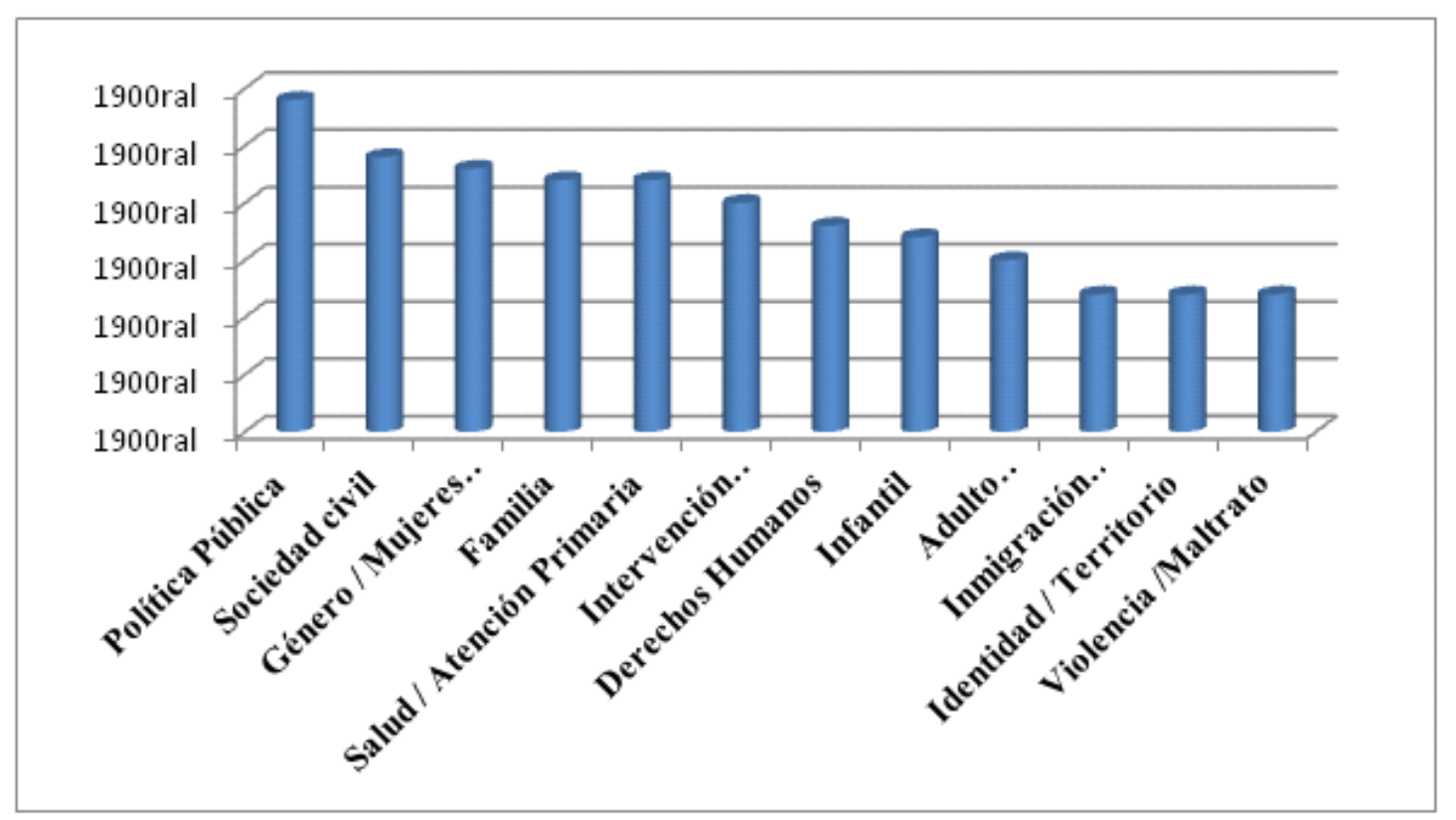

Fuente: Elaboración Propia a partir de Key Words de los 113 artículos revisados (2020).

\section{Consideraciones finales}

El trabajo desarrollado - consciente de sus limitaciones metodológicas ya que restringe la revisión de artículos publicados solo a revistas chilenas -abre una interesante perspectiva de análisis al acercarse cualitativamente y cuantitativamente a un fenómeno poco explorado en Chile como es la producción científica de los y las Trabajadores(as)Sociales en revistas especializadas. Los resultados indican una preferencia de los y las autoras hacia el paradigma interpretativo en sus líneas fenomenológicas o hermenéuticas, aunque suponemos que debiera profundizarse la distinción entre una y otra. El uso de técnicas de recolección de datos es mayoritariamente de tipo cualitativo por lo que sugerimos una mayor especialización en la dirección del entrenamiento de las entrevistas hacia el área investigativa. Es un tema interesante la alta producción de investigaciones desarrolladas mediante revisión bibliográfica $\mathrm{o}$ análisis documental, lo que es una herramienta significativa para los trabajadores sociales que ven limitadas sus posibilidades de investigación como queda establecido en esta investigación. Sería fundamental acercar a los (as) Trabajadores(as) sociales a software de análisis de programas como el Atlas Ti o el NVIVO, que permita mejorar aún más el análisis de los datos obtenidos. También es una tarea pendiente el revisar políticas que incentiven la investigación científica de profesionales no académicos pues son ellos los que están vinculados en primera línea con los distintos fenómenos sociales por lo que sería pertinente democratizar aún más este tipo de espacios científicos. Desde la vereda cuantitativa o positivista, se sugiere de igual modo el empleo de programas como el SPSS que permita el cruce de variables y la comprobación empírica de las hipótesis. Cualquiera sea el camino epistemológico o metodológico- claramente no es el propósito de este trabajo inclinarse en una u otra dirección. En este sentido, es fundamental fortalecer en los currículos de las licenciaturas ya que desde ellos se potencia la capacidad investigativa de los profesionales y se fundamenta el carácter disciplinar del Trabajo Social, como un campo de conocimiento propio en la complejidad.

Pareciera ser que el fortalecimiento de lo cualitativo debiera ir acompañado de mayor diversidad de metodologías en esta línea, disminuyendo entonces la hegemonía de asignaturas como estadísticas en los programas de estudio- ya que cada vez más irrumpen programas que buscan en lo interpretativo mejores formas de abordar la realidad por sobre su medición, cuantificación y neutralidad. También es interesante destacar los temas abordados por los y las Trabajadores (as) Sociales como es el caso de la políticas públicas, la preocupación por revisar las modalidades y supuestos de la intervención profesional. Sin duda será un gran paso el profundizar es estos aspectos -quizás por áreas - de manera de hacer viva una comunidad científica. Por otra parte, sería interesante poder conocer también quienes son los usuarios de estos conocimientos producidos en estas revistas y así de alguna manera conocer el impacto de la producción científica, y conocer por ejemplo en qué medida estos conocimientos fueron utilizados por los lectores, o como fueron utilizados estos resultados en otras investigaciones 
no solo en Chile sino también en otras latitudes. Se trata de construir comunidades científicas que aprendan colectivamente en sus materias. Solo así este tipo de producción tendrá sentido y no solo quedará almacenado en algún repositorio institucional. Finalmente, las políticas públicas de incentivo a la ciencia debieran sincerar sus perspectivas de cientificidad hacia las investigaciones cualitativas y otorgar un mayor lugar de interés a publicaciones o investigaciones ya que de lo contrario seguirá existiendo una brecha en esta materia. En pleno siglo XXI, los aportes de las ciencias sociales cobran nuevamente alto interés científico ya que las sociedades esta vez centradas en las debilidades del modelo neoliberal y del creciente individualismo que precariza la convivencia social, se necesita de nuevos conocimientos, de nuevas epistemologías, más locales, menos colonizadas de saberes y cuerpos teóricos, que entreguen comprensiones alternativas a todo aquello que no entra en la tradicional lógica científica. El desafío ahora es en que medida el grado de licenciatura deja de ser un accesorio formal y pasa a constituir una plataforma real y vigoroza que cree una verdadera comunidad científica.

\section{Referencias}

Arellano, N. \& Castro, B. (2017) Humanidades para el Trabajo Social y su Intervención: Apuesta por una Identidad "Descalzada". Revista Intervención, 7, 27-35.

Corbetta, P. (2007). Metodología y Técnicas de Investigación. Social. España: McGraw-Hill/Interamericana de España.

Diéguez, A. (2010). “QQué es la ciencia?”. En Diéguez, A. Filosofía de la Ciencia. Madrid: Biblioteca Nueva.

Enríquez, P. (2016). ¿Qué se enseña y cómo se enseña investigación en las escuelas de Trabajo Social?: Aproximaciones a la formación profesional de cuatro universidades de la Región Metropolitana. Revista Trabajo Social Pontificia Universidad Católica de Chile, 90. Habermas, J (1981) Teoría de la Acción Comunicativa. Racionalidad de la acción y racionalización social. Editorial Taurus Humanidades. Iturrieta, S. (2016). Entre Burbujas, sensaciones y realidades de la Profesión más masificada en Chile: El Trabajo Social. Revista Cuaderno de Trabajo Social, 9 (1), 9-26.

Linardelli, M. \& Pessolano, D. (2019). La Producción de conocimiento en Trabajo Social, una lectura desde las Epistemologías del sur y feministas. Revista de Trabajo Social e intervención social, 28.

Mardones, JMY \& Ursua N. (1982). Ciencias de la naturaleza y ciencias del espíritu. En Mardones, JMY \& Ursua N. Filosofía de las Ciencias Humanas y Sociales. Barcelona: Anthropos.

Mosquera, C. (2006). Conocimiento científico y saberes de acción en Trabajo Social: sobrevaloraciones, desconocimientos y revaloraciones: Una lectura desde los países de América del Norte. Revista del Departamento de Trabajo Social Universidad Nacional de Colombia, 8, 131-142.

Muñoz, G. (2018). Razón Neoliberal e Investigación: Resistencias desde el Trabajo Social. Cuadernos de Trabajo Social, 17. Padrón, J. (2007). Tendencias Epistemológicas de la Investigación Científica en el Siglo XXI. Revista Cinta de Moebio, 28 , 1-32. Toledo, U. (2004). ¿Una epistemología del trabajo social?. Cinta moebio, 21, 200-214.

\section{Christian Andrés Quinteros Flores}

cquinterosflores@gmail.com

Magister en Ciencia Politica, Universidad de Chile (UCH)

Docente Escuela de Gobierno y Gestiòn Pública Universidad de Chile (UCH)

\section{UCH}

Calle Huerfanos 1724

Santiago de Chile

CEP: 8340517

Agradecimentos

No aplica.

\section{Agência financiadora}

No aplica.
Consentimento para publicação

Consentimiento do autor.

Conflito de interesses

Não há conflito de interesses.

Aprovação por Comitê de Ética e consentimento para parti-

cipação

No aplica. 